\title{
Effects of Chronic Airway Inflammation on the Activity and Enzymatic Inactivation of Neuropeptides in Guinea Pig Lungs
}

\author{
Craig M. Lilly, * Lester Kobzik, *\$ Amy E. Hall, * and Jeffrey M. Drazen *\$ \\ ${ }^{*}$ Combined Program in Pulmonary and Critical Care Medicine, Departments of Medicine, Brigham and Women's Hospital and Beth \\ Israel Hospital; ${ }^{\ddagger}$ Department of Pathology, Brigham and Women’s Hospital and Harvard Medical School; and ${ }^{\S}$ Physiology Program, \\ Department of Environmental Health, Harvard School of Public Health, Boston, Massachusetts 02115
}

\begin{abstract}
The effects of airway inflammation induced by chronic antigen exposure on substance $P$ (SP)-induced increases and vasoactive intestinal peptide (VIP)-induced decreases in airway opening pressure (Pao), and the recovery of intact and hydrolyzed radiopeptide were studied in tracheally perfused guinea pig lungs. SP $\left(10^{-6} \mathrm{~mol} / \mathrm{kg}\right)$ induced a significantly greater increase in $\mathrm{Pao}$ in lungs from antigen-exposed $\left(30 \pm 5 \mathrm{~cm} \mathrm{H}_{2} \mathrm{O}\right)$ than saline-exposed animals $\left(15 \pm 1 \mathrm{~cm} \mathrm{H}_{2} \mathrm{O}, P<0.05\right)$. Significantly more intact ${ }^{3} \mathrm{H}-\mathrm{SP}$ and significantly less ${ }^{3} \mathrm{H}-\mathrm{SP}$ 1-7, a neutral endopeptidase (NEP) hydrolysis product, were recovered from the lung effluent of antigen-exposed than saline-exposed animals $(P<0.05)$. Injection of VIP $\left(10^{-9} \mathrm{~mol} / \mathrm{kg}\right)$ induced significantly more pulmonary relaxation in saline-exposed compared with antigen-exposed lungs $(62 \pm 4 \%, P$ $<0.001$ ). In contrast to effluent from saline-exposed animals, lung effluent from antigen-exposed lungs contained less intact VIP, increased amounts of a tryptic hydrolysis product, and no products consistent with the degradation of VIP by NEP. These data indicate that inflamed lungs are more sensitive to the contractile effects of SP because it is less efficiently degraded by NEP and are less sensitive to the relaxant effects of VIP because it is more efficiently degraded by a tryptic enzyme. Changes in airway protease activity occur with allergic inflammation and may contribute to airway hyperresponsiveness. ( $J$. Clin. Invest. 1994. 93:2667-2674.) Key words: vasoactive $\bullet$ intestinal peptide $\bullet$ substance $\mathbf{P} \bullet$ neutral endopeptidase $\bullet$ guinea pig • mast cell proteases $\bullet$ SCH 32,615
\end{abstract}

\section{Introduction}

Airway inflammation is now recognized as an important pathologic feature of asthma $(1,2)$. However, the mechanisms linking inflammation to the episodic airway obstruction and airway hyperresponsiveness characteristic of asthma have not been established. One possible link between airway inflammation and deranged physiological function is altered enzymatic inactivation of neuropeptides (3-6). In particular, there is reason to believe that altered inactivation of two peptides impor-

Address correspondence to Jeffrey M. Drazen, M.D., Combined Program in Pulmonary and Critical Care Medicine, Brigham and Women's Hospital, 75 Francis St., Boston, MA 02115.

Received for publication 2 September 1993 and in revised form 26 January 1994.

J. Clin. Invest.

(c) The American Society for Clinical Investigation, Inc.

$0021-9738 / 94 / 06 / 2667 / 08 \quad \$ 2.00$

Volume 93, June 1994, 2667-2674 tant for regulating airway responses, substance $\mathrm{P}(\mathrm{SP})^{1}$ and vasoactive intestinal peptide (VIP), could occur in the inflammatory microenvironment. Since the physiological effects of these peptides are known to be limited by enzymatic hydrolysis, it is possible that inflammation, by influencing the expression or activity of these enzymes, could alter hydrolytic inactivation of these peptides. SP is hydrolyzed and inactivated by neutral endopeptidase (NEP) (7-10) (E.C.3.4.24.11), while VIP is hydrolyzed and inactivated by NEP and a tryptic enzyme (11). If in the inflammatory microenvironment NEP activity is diminished, the physiological effects of SP would be enhanced. However, the effects of inflammation on VIP activity will depend on alterations in both NEP and tryptic activity. To determine the importance of airway inflammation induced by chronic antigen exposure on the activity and enzymatic inactivation of SP and VIP, we examined the physiological effects, recovery of intact peptide, and recovery of the hydrolysis products of SP and VIP in normal and chronically inflamed guinea pig lungs. We found that lungs with chronic allergic inflammation were more sensitive to the contractile effects of SP and less sensitive to the relaxant effects of VIP than control lungs. In addition, the effects of enzyme inhibitors on physiological responses and peptide cleavage profiles were consistent with decreased NEP and enhanced tryptic activity.

\section{Methods}

Antigen exposure protocol. Airway inflammation was accomplished by ovalbumin exposure and sensitization modified from the method of Hutson et al. $(12,13)$. Male Hartley guinea pigs, $300-350 \mathrm{~g}$ body weight, were pretreated with pyrilamine malate $10 \mathrm{mg} / \mathrm{kg}$ (doses are expressed per $\mathrm{kg}$ animal) given by intraperitoneal injection $10 \mathrm{~min}$ before each aerosol exposure; the pyrilamine prevented respiratory distress secondary to the release of histamine during the sensitization period. Animals were sensitized by exposure to aerosolized ovalbumin ( $1 \% \mathrm{wt} / \mathrm{vol}$ in $0.9 \%$ sterile sodium chloride) or saline alone in an aerosol exposure chamber which exposed six animals simultaneously to a single aerosolized stream. Each animal had its snout fixed $15 \mathrm{~cm}$ from the point of aerosol entry in a chamber with a volume of 33 liters. Nebulization was accomplished by two Acorn $1^{\oplus}$ nebulizers (Marquest Medical Products Inc., Englewood, $\mathrm{CO}$ ), which were powered by pressurized oxygen delivered at a flow rate of 8 liters/min. After a 3-min exposure period, the chamber was cleared of aerosol by vacuum suction through a filter apparatus and room air was provided to the animals. Animals were exposed on three occasions at 7-d intervals, and the lungs were harvested three days after the final exposure.

Histopathologic examination. Two groups of five male Hartley strain guinea pigs ( $330-410 \mathrm{~g}$ ) repeatedly exposed to either antigen or saline aerosol as described above, were anesthetized with $65 \mathrm{mg}$ sodium

1. Abbreviations used in this paper: NEP, neutral endopeptidase; Pao, airway opening pressure; SP, substance P; VIP, vasoactive intestinal peptide. 
pentobarbital $/ \mathrm{kg}$ given by intraperitoneal injection. When an appropriate plane of anesthesia was achieved, the abdominal cavity was opened and $500 \mathrm{U}$ of heparin were injected into the vena cava. $3 \mathrm{~min}$ later the abdominal aorta was severed and the guinea pig was exsanguinated. The thoracic cavity was opened and a right ventricular catheter was placed. Karnovsky's fixative was infused into the catheter at $20 \mathrm{~cm}$ $\mathrm{H}_{2} \mathrm{O}$ pressure. After lung expansion and equilibration the heart-lung bloc was removed. The distal $1 \mathrm{~cm}$ of trachea was bisected longitudinally and a coronal section of the right lower lobe, including the proximal bronchovascular bundle were placed in Karnovsky's fixative overnight and stored at $4^{\circ} \mathrm{C}$ for at least $24 \mathrm{~h}$ in cacodylate buffer. Fixed tissues were embedded in glycolmethacrylate, sectioned, and stained with methylene blue and basic fuchsin.

The degree of airway inflammation was quantitated by a pulmonary pathologist blinded as to the exposure status of the animals from which the lungs were harvested. Histologic grading of airway inflammation, from the trachea to the lobar bronchi, was accomplished by examining the airway epithelium at $\times 400$ and scoring the presence of inflammatory cells semi-quantitatively as presented in Table $I(12,13)$. The percentage of mast cells, in the trachea, demonstrating histologic evidence of degranulation was calculated from counts of all mast cells present in a six tracheal ring span of coronally cut trachea. In addition to the 10 lungs specifically prepared for histologic study, tracheal and parenchymal sections of all tracheally perfused lungs were fixed in Karnovsky's fixative and subjected to histologic evaluation as described above.

Tracheally perfused lung preparation. Tracheal perfusion was performed as previously described $(9,11): 112$ male Hartley strain guinea pigs ( 57 saline-exposed and 55 antigen-exposed), (330-410 g) were anesthetized with $65 \mathrm{mg}$ of sodium pentobarbital $/ \mathrm{kg}$ by intraperitoneal injection. When an appropriate plane of anesthesia was achieved, a tracheostomy was created and a 2-cm length of polyethylene tubing ( $1.67 \mathrm{~mm}$ I.D., $2.42 \mathrm{~mm}$ O.D.) was placed in the trachea. The abdominal cavity was opened and $500 \mathrm{U}$ of heparin were injected into the vena cava. 3 min later the abdominal aorta was severed and the guinea pig was exsanguinated. The thoracic cavity was widely opened and the heart and lungs were removed en bloc. The lungs were dissected free and placed in a $37^{\circ} \mathrm{C}, 100 \%$ relative humidity Plexiglas ${ }^{\oplus}$ box. The lungs were tracheally perfused with a phosphate-buffered physiological solution whose composition was $\mathrm{NaCl} 137 \mathrm{mM}, \mathrm{CaCl}_{2} 1.8 \mathrm{mM}, \mathrm{MgCl}_{2}$ $1.05 \mathrm{mM}, \mathrm{KCl} 2.68 \mathrm{mM}, \mathrm{NaHCO}_{3} 0.6 \mathrm{mM}, \mathrm{NaH}_{2} \mathrm{PO}_{4} 0.13 \mathrm{mM}$, and $\mathrm{Na}_{2} \mathrm{HPO}_{4} 0.896 \mathrm{mM}, \mathrm{pH}$ 7.4. The perfusion buffer was warmed to $45^{\circ} \mathrm{C}$ and pumped at $5 \mathrm{ml} / \mathrm{min}$ through a bubble trap before being cooled to $37^{\circ} \mathrm{C}$ and administered to the lungs through the tracheal cannula. Perfusate exited the fully expanded lungs via numerous small holes placed in the pleura. The "back pressure" resulting from tracheal perfusion (airway opening pressure, $\mathrm{Pao}$ ) was recorded from a side tap at the tracheal cannula with the use of a pressure transducer (P23Db; Statham Instruments Inc., Oxnard, CA). We have previously shown that during continuous flow Pao reflects the contractile state of the lung $(9,11)$. All experiments involving animals were approved by the appropriate animal care and use committees.

Table I. Scoring System for Histologic Grading of Airway Inflammation

\begin{tabular}{cl}
\hline Score & \multicolumn{1}{c}{ Appearance of airways } \\
\hline 0 & No inflammatory cells seen \\
1 & Occasional granulocytes in some but not all fields \\
2 & Granulocytes present in every field \\
3 & Abundant granulocytes ( $>10 /$ high powered field) \\
& present in every field examined
\end{tabular}

Protocols for comparing SP and methacholine effects in inflamed and normal lungs

Effects of inflammation on SP- or methacholine-Pao dose-response relationship. SP was rapidly injected into the perfusion stream of tracheally perfused lungs harvested from antigen-exposed and saline-exposed animals, and the resulting increase in Pao was recorded. Pao responses were recorded for 10 groups of three lungs each ( 15 antigenexposed and 15 saline-exposed), injected with geometrically increasing doses of SP $\left(3 \times 10^{-8}\right.$ to $\left.3 \times 10^{-6} \mathrm{~mol} / \mathrm{kg}\right)$. A single measurement was obtained per lung. Pao responses were recorded for two groups of five lungs each (five antigen-exposed and five saline-exposed), injected with geometrically increasing doses of methacholine.

Effects of NEP inhibition on SP-induced Pao responses in inflamed lungs. Pao was recorded after tracheal injection of $10^{-6} \mathrm{~mol} \mathrm{SP} / \mathrm{kg}$ in four groups of five tracheally perfused lungs. Two of these groups were antigen-exposed and two groups were saline-exposed. SCH 32,615 was dissolved in DMSO and diluted 1:10,000 into the perfusion buffer to yield a final concentration of $10^{-6} \mathrm{M}$. An equal amount of DMSO was added to the perfusion buffer of the groups that did not receive SCH 32,615 .

Effects of inflammation on SP hydrolysis product profile. ${ }^{3} \mathrm{H}-\mathrm{SP}$ $\left(10^{-6} \mathrm{dpm}\right)$ and $3 \times 10^{-7}$ moles of nonradiolabeled SP was injected into the perfusion stream of three antigen-exposed tracheally perfused lungs and five saline-exposed lungs. Lung effluent was collected as previously described (9); the lung effluent fraction containing SP cleavage products (2.5-5 min after SP injection) was subjected to HPLC analysis as described below.

Identification of ${ }^{3} \mathrm{H}-S P$ and ${ }^{3} \mathrm{H}-\mathrm{SP}$ hydrolysis products. Identification of potential SP cleavage products (i.e., SP 1-6, SP 1-7, and SP 1-9) was accomplished by comparing the reverse-phase HPLC retention times of synthetic SP and its peptide fragments to the retention times of ${ }^{3} \mathrm{H}$-radiolabel recovered from lung effluent. Reverse-phase HPLC separation was accomplished with the use of $\mathrm{C}_{18}$ reverse-phase column (Nova-Pak ${ }^{\oplus}, 3.9 \times 300$ mm; Waters Instruments, Milford, MA) at a flow rate of $1 \mathrm{ml} / \mathrm{min}$. The initial mobile phase was HPLC grade $\mathrm{H}_{2} \mathrm{O}$ with $0.1 \%$ trifluroacetic acid (TFA); the column was eluted with a $0.7 \%$ to $49 \%$ linear gradient of acetonitrile in HPLC grade $\mathrm{H}_{2} \mathrm{O}$ with $0.1 \%$ TFA $>40 \mathrm{~min}$. The ${ }^{3} \mathrm{H}$ content was determined by scintillation counting (Beckman Instruments Inc., Fullerton, CA) aliquots of HPLC effluent collected at 1-min intervals and suspended in scintillation counting solution (National Diagnostics, Mannville, NJ). Peptide loss to the column was $<5 \%$.

\section{Protocols for comparing the relaxant effects of VIP in inflamed and normal lungs}

Effects of inflammation on the relaxant response to VIP. During tracheal perfusion with $10^{-7} \mathrm{M}$ methacholine in the perfusion buffer, Pao rose slowly and reached a stable plateau between 13 and $18 \mathrm{~min}$. In this setting tracheal injection of VIP or isoproterenol caused a rapid decrease in Pao; all data examining pulmonary relaxation are reported in lungs with methacholine-induced airway tone. VIP was rapidly injected into the perfusion stream of tracheally perfused lungs harvested from animals exposed to antigen or saline, and the resulting decrease in $\mathrm{Pao}$ was recorded. Two groups of five lungs each were exposed to geometrically increasing doses of VIP $\left(10^{-10}-10^{-7} \mathrm{~mol} / \mathrm{kg}\right)$, and Pao responses were recorded. Two groups of five lungs each were also exposed to geometrically increasing doses of isoproterenol $\left(10^{-11}-10^{-8} \mathrm{~mol} /\right.$ $\mathrm{kg}$ ), and the Pao response was recorded.

Effects of inflammation on VIP recovery in lung effluent. After tracheal injection of $10^{-9}$ moles of VIP, a 5-min fraction of lung effluent was collected in $5 \%$ by volume glacial acetic acid, dried under vacuum and stored at $-20^{\circ} \mathrm{C}$. Recovery of VIP was quantified by an ELISA, which is insensitive to VIP hydrolysis products, as previously described (11).

Effects of inflammation on VIP hydrolysis product profile. After tracheal injection of $250,000 \mathrm{dpm}$ of (mono [ $\left.{ }^{125} \mathrm{I}\right]$ iodo-Tyr $\left.{ }^{10}\right)-\mathrm{VIP}$ 
and $10^{-9} \mathrm{~mol}$ of nonradiolabeled VIP, serial 1-min fractions of perfusate were recovered from lungs harvested from animals that had been exposed to antigen $(n=2)$, from lungs harvested from animals exposed to saline $(n=2)$, and from lungs treated with SBTI, $500 \mathrm{TIU} / \mathrm{kg}$, harvested from animals exposed to antigen $(n=2)$. Samples were dried under vacuum, resuspended in $600 \mu \mathrm{l}$ of HPLC grade $\mathrm{H}_{2} \mathrm{O}$ with $0.1 \%$ ( vol/vol) TFA, divided into two 300- $\mu \mathrm{l}$ aliquots, passed through a $0.22-\mu \mathrm{m}$ filter (Millipore Corp., Medford, MA), and subjected to RPHPLC analysis as described below. Recovery of radiolabel was $>90 \%$.

Effects of inflammation on inhibitor (SBTI) potentiation of VIP-induced pulmonary relaxation. Pao was recorded after tracheal injection of $10^{-9} \mathrm{~mol} / \mathrm{kg}$ VIP in four groups of five tracheally perfused lungs. Two of these groups were antigen-exposed and two were saline exposed. The "antigen-exposed + SBTI" and "saline-exposed + SBTI" groups had $500 \mathrm{TIU} / \mathrm{kg}$ SBTI added to their perfusion buffer.

Preparation of (Mono $\left[{ }^{125} I\right]$ iodo-Tyr $\left.{ }^{10}\right)-V I P$ and $\left(\left[{ }^{125} I\right]\right.$ iodo$\left.T y r^{10}\right)$-VIP fragments. VIP or VIP fragments were iodinated by the method of Martin et al. (14). $1.5 \times 10^{-9} \mathrm{~mol}$ of VIP or a VIP fragment was dissolved in $25 \mu \mathrm{l}$ of $0.3 \mathrm{M} \mathrm{PBS}, \mathrm{pH} 7.6$, and $1 \mathrm{mCi}$ of carrier-free sodium iodide $(0.5 \mathrm{nmol}$ in $10 \mu \mathrm{l} \mathrm{NaOH}, \mathrm{pH} 7.4$; Amersham International, Amersham, UK) was added, providing $\sim 3$ molecules of VIP or VIP fragment for each ${ }^{125}$ I-atom. During stirring, iodination was initiated with the addition of $14.2 \mathrm{nmol}$ of chloramine- $\mathrm{T}$ at a concentration of $1 \mathrm{mg} / \mathrm{ml}$. After $15 \mathrm{~s}$ at room temperature, the reaction was terminated by the addition of $42.1 \mathrm{nmol}$ of $\mathrm{Na}_{2} \mathrm{~S}_{2} \mathrm{O}_{5}$. The reaction mixture was applied to a Sep-Pak ${ }^{\otimes} \mathrm{C}_{18}$ cartridge and washed with $9 \mathrm{ml}$ of HPLC grade $\mathrm{H}_{2} \mathrm{O}$ with $0.1 \%$ TFA. ${ }^{125} \mathrm{I}$-VIP or ${ }^{125} \mathrm{I}$-VIP fragment was eluted in a solution of $60 \%$ acetonitrile and $40 \% \mathrm{H}_{2} \mathrm{O}$ with $0.1 \%$ TFA, pH 2.5 . The eluate was purified by $\mathrm{C}_{18}$ reverse-phase chromatography with the RP-HPLC protocol described below as Protocol 1. Two sequential HPLC purification steps yielded a single peak with a reproducible retention time. Purified material was stored at $4^{\circ} \mathrm{C}$ and was used the following day.

Identification of VIP and VIP hydrolysis products. Identification of potential VIP cleavage products (i.e., VIP 1-22, VIP 1-21, VIP 1-14, VIP 4-14, VIP 5-14, VIP 5-21, VIP 5-22, VIP 5-28, VIP 9-10, VIP 10-11, VIP 21-22, VIP 22-23, and VIP 22-28) was accomplished by comparing the RP-HPLC retention time of authentic ${ }^{125} \mathrm{I}$-VIP and iodinated synthetic fragments to the retention time of ${ }^{125}$ I-radiolabel recovered from lung effluent in two distinct RP-HPLC protocols. RPHPLC separation was accomplished with the use of $\mathrm{C}_{18}$ reverse-phase column (Nova-Pak ${ }^{\circledR}, 3.9 \times 300 \mathrm{~mm}$ (Waters Instruments) at a flow rate of $1 \mathrm{ml} / \mathrm{min}$. The initial mobile phase for both HPLC protocols was $\mathrm{HPLC}$ grade $\mathrm{H}_{2} \mathrm{O}$ with $0.1 \%$ TFA. In elution protocol 1 , the column was eluted with a $0.7-49 \%$ linear gradient of acetonitrile over 40 min. In elution protocol 2 , the column was eluted with a $14-49 \%$ linear gradient of acetonitrile over $55 \mathrm{~min}$. 30-s fractions were collected, and ${ }^{125}$ I content was determined by gamma counting (Packard, Sterling, VA). Authentic standards for all peptides were processed through the system without a lung present, yielding recoveries of 33-95\%; radiochromatograms were corrected for the background activity and for losses due to processing. The inhibitor combinations used had no effect on the elution time of the VIP breakdown products.

Materials. VIP (guinea pig) 1-28, VIP fragments 1-21, 5-14, 5-21, 5-22, 5-28, 9-10, 10-11, 21-22, 22-23, 22-28, SP 1-11, SP 1-6, SP 1-7, SP 1-9, VIP (guinea pig), and SP antisera were obtained from Peninsula Laboratories (Belmont, CA). (2-prolyl-3,4- ${ }^{3} \mathrm{H}[\mathrm{N}]$ )-SP was obtained from DuPont NEN ${ }^{\circledR}$ Research Products (Wilmington, DE). VIP fragments 1-22, 1-14, and 5-22 were custom synthesized by Hospital Peptide Services (Boston, MA). VIP 4-14 was custom synthesized by Research Genetics (Huntsville, AL). Glutaraldehyde, soybean trypsin inhibitor, DMSO, isoproterenol, chloramine-T, metabisulfite, pyrilamine, and methacholine were obtained from Sigma Chemical Co. (St. Louis, MO). SCH 32,615 was the kind gift of Schering Pharmaceuticals (Kenilworth, NJ). All materials were HPLC or reagent grade as appropriate.

Statistical analysis. All values are expressed as mean \pm standard error of the mean (SEM), unless otherwise stated. The statistical signifi- cance of differences between means was determined by analysis of variance (ANOVA); $P<0.05$ was considered significant. When ANOVA indicated a significant difference, the Newman-Keuls test was used to determine which groups were significantly different from each other. In the histological study and the studies of neuropeptide effects, data from antigen-exposed animals and saline-exposed animals receiving equal doses of neuropeptide were compared by the Student's $t$ test.

\section{Results}

Histopathologic examination. Repeated exposure to aerosol ovalbumin was effective in inducing airway inflammation (Fig. 1). The histologic grade of airway inflammation was $2.1 \pm 0.1$ in antigen-exposed lungs, which was significantly greater than that of saline-exposed lungs $(0.6 \pm 0.2 ; P<0.001 ; n$ $=5$ ). A histologic grade of $>2$ indicated that inflammatory cells could be seen in nearly every field examined from the trachea to the lobar bronchi, while a grade $<1$ indicated that few inflammatory cells were present. Tracheal sections and airways present in parenchymal sections of antigen-exposed, but not saline-exposed, animals demonstrated mucosal and submucosal infiltration most prominently with eosinophils, but neutrophils and occasional lymphocytes were also present. Abnormalities were not observed in the lung parenchyma. The percentage of mast cells demonstrating histologic evidence of degranulation was significantly greater in the tracheas of antigen exposed compared to saline exposed animals $(44 \pm 14 \%$ compared to $9 \pm 8 \%, P<0.001$ ).

Effects of inflammation on SP-Pao and methacholine doseresponse relationship. Antigen-exposed lungs were significantly more responsive to the contractile effects of SP at doses from $3 \times 10^{-7}$ to $3 \times 10^{-6} \mathrm{~mol} / \mathrm{kg}(P<0.05, n=3)$ than saline-exposed lungs, Fig. 2. Antigen-exposed lungs were also significantly more responsive to the contractile effects of methacholine. The methacholine $\mathrm{ED}_{50}$ was $8.6 \pm 0.6 \times 10^{-8}$ moles in antigen exposed lungs compared to $6.4 \pm 1.1 \times 10^{-7}$ moles in saline exposed lungs $(P<0.01, n=5)$.

Effects of NEP inhibition on SP-induced Pao responses in inflamed lungs. Tracheal injection of $10^{-6} \mathrm{~mol} / \mathrm{kg}$ SP into lungs from saline-exposed animals resulted in an increase in $\mathrm{PaO}$ of $15 \pm 1 \mathrm{~cm} \mathrm{H} \mathrm{H}_{2} \mathrm{O}(n=5)$. When the NEP inhibitor $\mathrm{SCH}$ $32,615\left(10^{-6} \mathrm{M}\right)$ was added to the perfusion buffer of lungs from saline-exposed animals, a significant potentiation of the contractile effects of SP was observed; Pao increased $41 \pm 5 \mathrm{~cm}$ $\mathrm{H}_{2} \mathrm{O}(P<0.005$ compared with no SCH 32,615, $n=5)$ (Fig. $3)$. In lungs from antigen-exposed animals, Pao increased $30 \pm 5 \mathrm{~cm} \mathrm{H}_{2} \mathrm{O}(n=5)$ after tracheal injection of an identical amount of SP. This response was significantly greater than that observed in lungs from saline-exposed animals not treated with the NEP inhibitor $(P<0.05)$ but not significantly different from that of lungs from saline-exposed animals treated with the NEP inhibitor. In contrast, the NEP inhibitor did not affect the $\mathrm{Pao}$ response to SP in lungs from antigen-exposed animals. Pao increased $35 \pm 6 \mathrm{~cm} \mathrm{H}_{2} \mathrm{O}$ in lungs from the antigen-exposed group treated with $\mathrm{SCH} 32,615$; this response was not signifcantly different from that observed in lungs from antigen-exposed animals not treated with the NEP inhibitor $(30 \pm 5 \mathrm{~cm}$ $\mathrm{H}_{2} \mathrm{O}, P=$ NS. $n=5$ ) or in lungs from the saline-exposed group treated with $\mathrm{SCH} 32,615$. The Pao response in antigen-exposed animals was significantly greater than the Pao response in saline-exposed animals not treated with the NEP inhibitor ( $P$ $<0.05$, Fig. 3). 

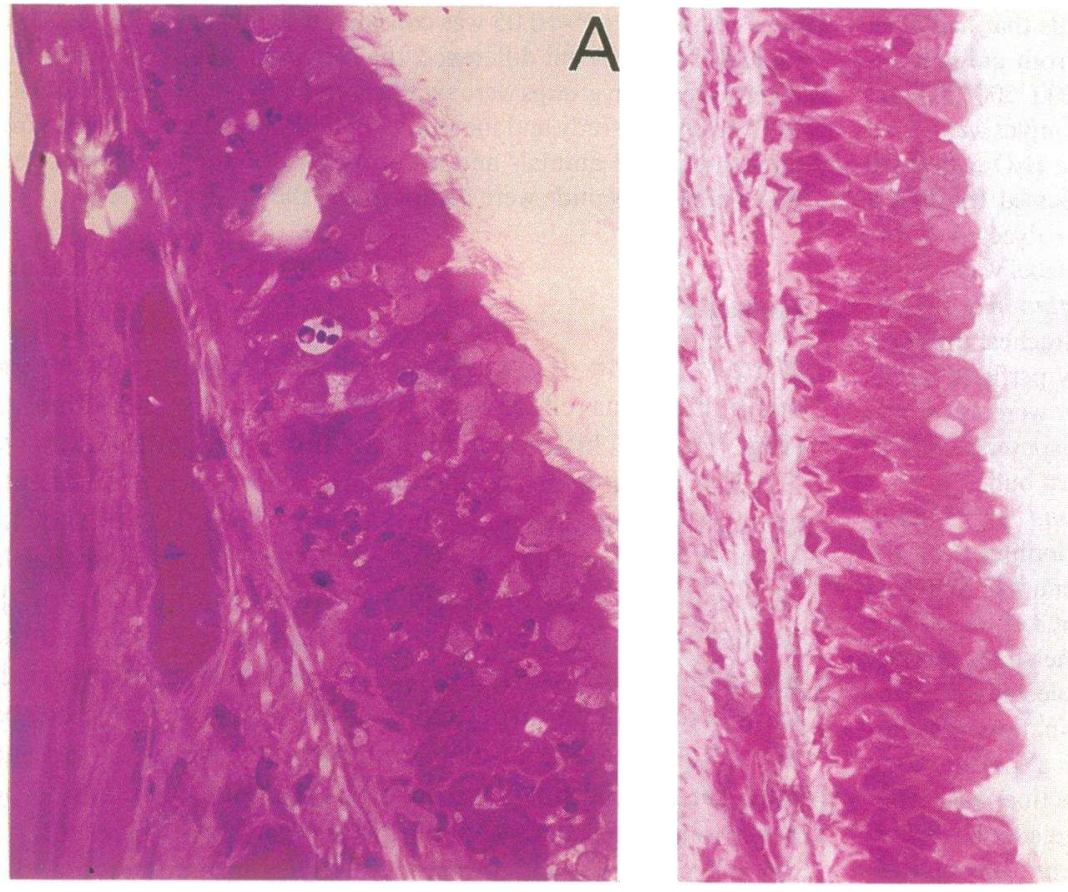

Figure 1. The histologic grade of airway inflammation was significantly greater in antigen-exposed $(A)$ compared with salineexposed $(B)$ lungs $(P<$ $0.001, n=5)$. Fixed tissues were stained with methylene blue and basic fuchsin $(\times 400)$.

Effects of inflammation on SP hydrolysis product profile. Analysis of effluent collected from the lungs removed from saline-exposed animals after tracheal injection of ${ }^{3} \mathrm{H}-\mathrm{SP}$ revealed intact SP and SP fragments corresponding to NEP hydrolysis sites (i.e., SP 1-9, SP 1-7, and SP 1-6) $(n=5$; Fig. 4 B). In contrast, analysis of effluent collected from the lungs of antigen-exposed animals after tracheal injection of ${ }^{3} \mathrm{H}$-SP revealed a significantly greater amount of intact $\mathrm{SP}$ and a lesser amount of the NEP hydrolysis product, SP 1-7 $(P<0.05, n=3$; Fig. $4 A$ ).

Effects of inflammation on the relaxant response to VIP. Lungs removed from antigen-exposed animals were resistant to the relaxant effects of VIP. Pao decreased $37 \pm 13 \%$ after tracheal injection of $10^{-10} \mathrm{~mol} / \mathrm{kg}$ VIP and $62 \pm 4 \%$ after injection

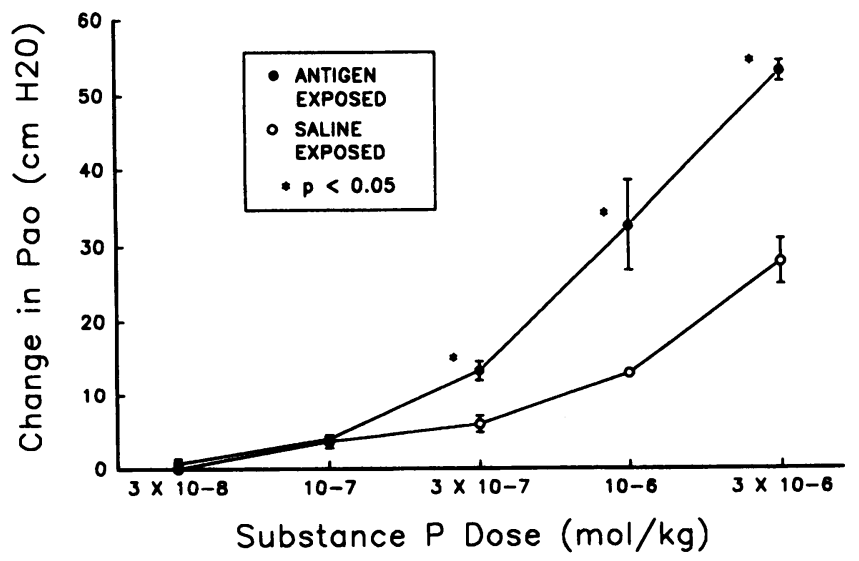

Figure 2. SP-Pao dose-response relationships were measured in antigen-exposed and saline-exposed lungs. Tracheally perfused lungs from antigen-exposed animals $(\bullet)$ were significantly more sensitive to the contractile effects of SP than lungs from saline-exposed (O) animals $(P<0.05, n=3)$. of $10^{-9} \mathrm{~mol} / \mathrm{kg}$ into the lungs of saline-exposed animals-each dose of VIP resulted in a significant decrease in Pao, while there was no Pao effect from these doses in lungs from antigen-exposed animals $\left(P<0.05\right.$ for $10^{-10} \mathrm{~mol} / \mathrm{kg}$ and $P<0.001$ for $10^{-9} \mathrm{~mol} / \mathrm{kg}, n=5$, Fig. 5). When $10^{-8} \mathrm{~mol} / \mathrm{kg}$ VIP was administered, Pao decreased $48 \pm 13 \%$ in lungs from antigenexposed animals, while Pao decreased $89 \pm 5 \%$ in lungs from saline-exposed animals $(P<0.05, n=5)$. There was no significant difference in the responsiveness of lungs from antigen-exposed and saline-exposed animals to the relaxant effects of isoproterenol ( $P=\mathrm{NS}, n=5$, Fig. 5).

Effects of inflammation on VIP recovery from lung effluent. After tracheal injection of $10^{-9}$ moles of VIP, significantly less unhydrolized VIP was detected in the effluent of antigen exposed compared to saline exposed lungs $(65 \pm 17$ pmol compared to $200 \pm 8$ pmol, $P<0.001$ ).

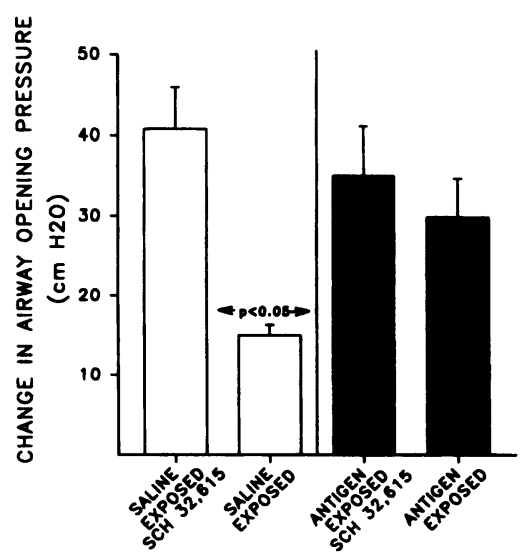

Figure 3. The effects of NEP inhibition on SR induced increases in Pao were compared in groups of lungs from antigen-exposed and saline-exposed animals. SP contractile effects were augmented by the NEP inhibitor SCH 32,615 in lungs from saline-exposed animals ( $n=5, P<0.05$ for the saline-exposed group compared to all other groups). In contrast, $\mathrm{SCH}$ 32,615 had no significant effect in lungs from antigen-exposed animals $(P=\mathrm{NS}, n=5)$. 
A ANTIGEN EXPOSED

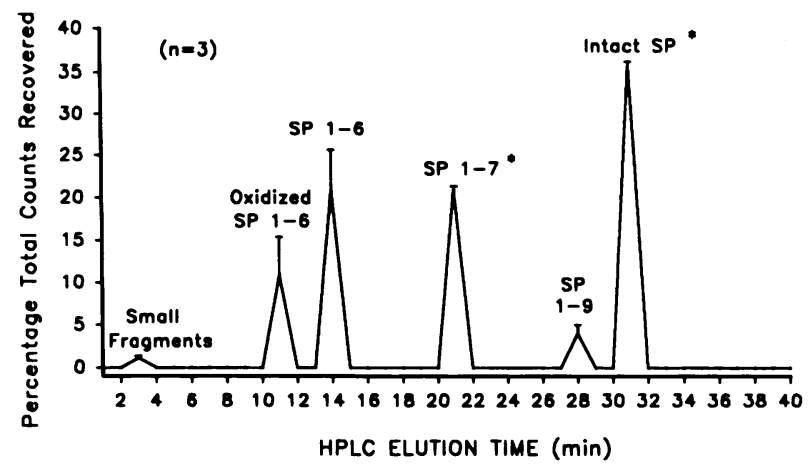

B SALINE EXPOSED

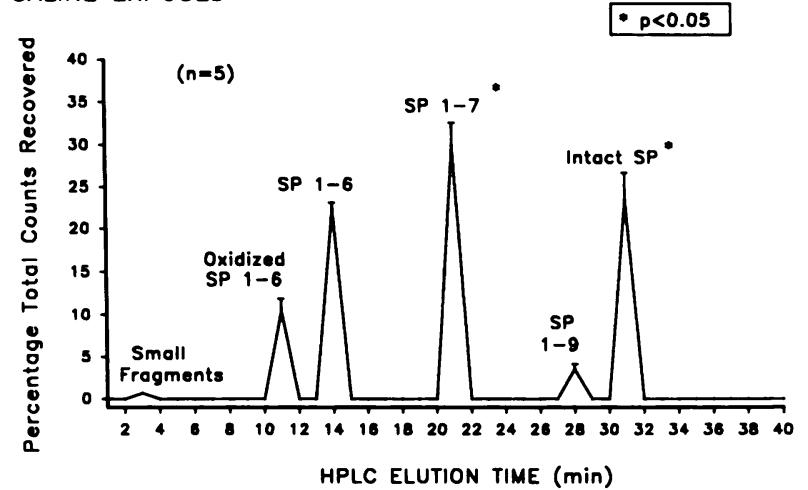

Figure 4. The recovery of intact ${ }^{3} \mathrm{H}-\mathrm{SP}$ and ${ }^{3} \mathrm{H}-\mathrm{SP}$ hydrolysis fragments was measured in lung effluent from antigen-exposed and saline-exposed animals. Recovery of intact ${ }^{3} \mathrm{H}-\mathrm{SP}$ in the lung effluent of antigen-exposed animals $(A)$ was greater than the recovery from lung effluent of saline-exposed animals $(B)$. The recovery of ${ }^{3} \mathrm{H}-\mathrm{SP}$ 1-7, a hydrolysis product of NEP activity, was less from effluent of lungs from antigen-exposed $(n=3)$ than saline-exposed animals ( $P$ $<0.05, n=5$ ).

Effects of inflammation on VIP hydrolysis product profile. There were fewer VIP hydrolysis products consistent with NEP activity in the effluent of tracheally perfused lungs removed from antigen-exposed compared with saline-exposed animals. $28 \%$ of the radiolabel coeluted with intact VIP 1-28 in the effluent of lungs from saline-exposed animals, while only $12 \%$ of the radiolabel coeluted with VIP 1-28 in the effluent from the lungs of antigen-exposed animals. In lung effluent from salineexposed animals, only $39 \%$ of the radiolabel coeluted with VIP $1-14$, while $88 \%$ of the radiolabel administered to antigen-exposed lungs coeluted with this fragment of tryptic hydrolysis. In lungs from saline-exposed animals, 33\% of the radiolabel coeluted with VIP 4,5-14, a product of combined hydrolysis by NEP and a tryptic enzyme. In contrast, we were unable to identify VIP hydrolysis products consistent with NEP activity in the effluent of antigen-exposed lungs (Fig. 6, representative data from single lungs). Examination of the VIP hydrolysis profile when SBTI was present in the perfusion buffer of lungs from antigen-exposed animals, reveals that more active, unhydrolized VIP and less inactive VIP 1-14 were recovered.

Effects of inflammation on inhibitor (SBTI) potentiation of $V I P$-induced pulmonary relaxation. Tracheal injection of $10^{-9}$ $\mathrm{mol} / \mathrm{kg}$ VIP into lungs of antigen-exposed animals resulted in a $3 \pm 2 \%$ decrease in Pao $(P<0.001$ compared with all other groups, $n=5$, Fig. 7). In the presence of SBTI, VIP relaxant activity was restored in lungs from antigen-exposed animals; Pao decreased by $57 \pm 2 \%$ in the "antigen-exposed + SBTI" group. Lungs from saline-exposed animals had a significant decrease in Pao after VIP injection. The Pao response to VIP injection was not altered by the presence of SBTI in saline-exposed lungs (Fig. 7).

\section{Discussion}

It is now well established that the physiological effects of the neuropeptides are regulated by their enzymatic degradation in the pulmonary microenvironment $(7-11,15-17)$. When SP is presented to the airway surface, its action is regulated predominantly by the activity of NEP, while the physiological effects of VIP are regulated by NEP and a tryptic enzyme. In each case the degradative enzymes are positioned such that they are physiologically competitive with each other and the relevant receptors $(18,19)$. It is also now well appreciated that the proteolytic capacity of the microenvironment can be altered by mediators of inflammation $(20,21)$. However, the mechanistic links among inflammation, altered enzyme activity, and abnormal airway responses have not been clearly elucidated. In the experiments reported herein, we demonstrate that recurrent antigen exposure is associated with an enhanced response to SP and a diminished response to VIP. Our data provide a potential

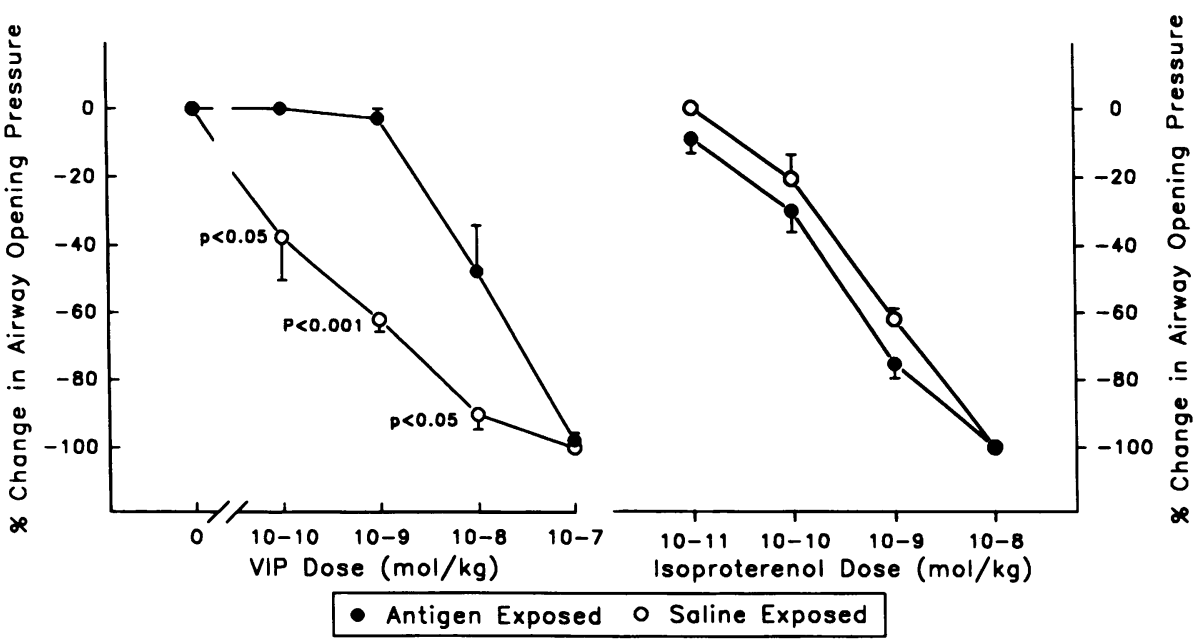

Figure 5. The relaxant effects of VIP and isoproterenol were compared in lungs from antigen-exposed and salineexposed animals. Lungs from antigenexposed animals were less responsive to the relaxant effects of VIP than lungs from saline-exposed animals $(n=5, P$ $<0.05)$. In contrast, there was no significant difference in the relaxant response to isoproterenol in lungs from antigen-exposed compared with salineexposed animals $(P=\mathrm{NS}, n=5)$. 

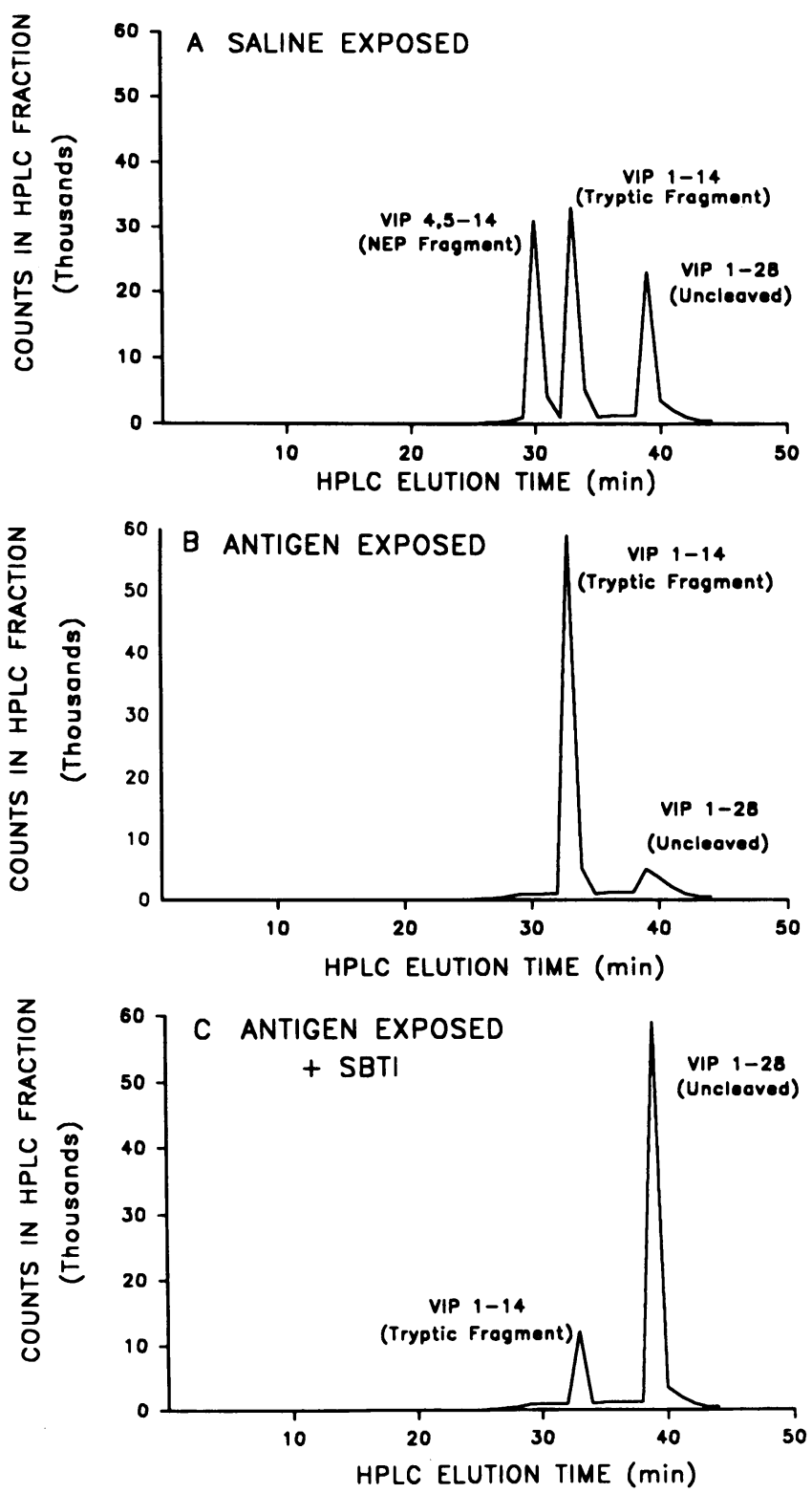

Figure 6. VIP hydrolysis product profile was compared in lungs from antigen-exposed and saline-exposed animals injected with ${ }^{125} \mathrm{I}$-VIP. VIP hydrolysis products consistent with activity by NEP and a tryptic enzyme were recovered from the lung effluent of saline-exposed animals injected with ${ }^{125} \mathrm{I}$-VIP $(A)$. In contrast, lung effluent from antigen-exposed animals $(B)$ contained less intact VIP and greater quantities of the product consistent with tryptic activity. Products consistent with NEP activity were recovered only in the lung effluent of saline-exposed animals. $(C)$ In antigen-exposed lungs treated with SBTI, less of the tryptic fragment and more intact VIP was recovered.

mechanism by which a chronic inflammatory microenvironment could alter airway responsiveness.

Our data demonstrate that the presence of allergic inflammation is associated with increased sensitivity of lungs to the contractile effects of SP (Fig. 2) and methacholine; these findings are in concert with the work of others $(12,13)$. Our data also suggest that the increased sensitivity to SP is the result of decreased hydrolytic inactivation of this peptide by NEP, (Fig. 3). Our ability to augment SP-induced pulmonary contraction in normal lungs is consistent with the predominant role of NEP

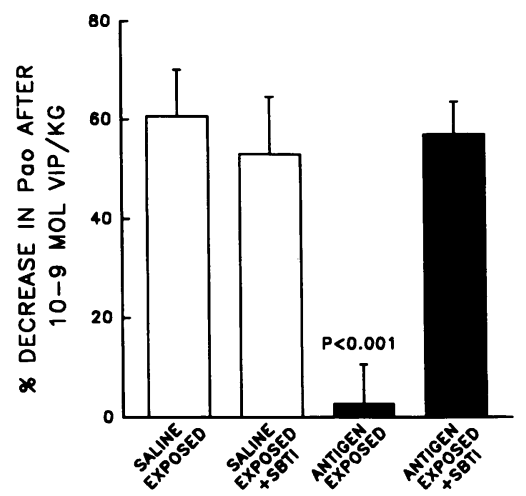

Figure 7. The effects of SBTI, an inhibitor of tryptic proteases, on VIP-induced pulmonary relaxation was measured in lungs from antigen-exposed and saline-exposed animals. SBTI augmented VIPinduced pulmonary relaxation in lungs from antigen-exposed animals $(P<0.001, n=5)$ but had no significant effect in lungs from saline-exposed animals $(P$ $=$ NS, $n=5$ ).

for regulating the airway contractile effects of SP when it is presented to the airway epithelial surface $(9,15,22-26)$. The observation that lungs removed from animals repeatedly exposed to antigen are not responsive to inhibitors of NEP implies that the fraction of SP responsible for airway obstruction is less efficiently inactivated by NEP in these lungs. According to our current understanding, loss of NEP inactivation of SP should lead to higher local concentrations of SP in the microenvironment and increased SP contractile effects. Indeed loss of NEP activity has been associated with enhanced SP contractile effects after TDI exposure (27), exposure to cigarette smoke (28), exposure to oxidants $(20,21,29,30)$, and airway infection by viral (31) or mycoplasmal pathogens (32).

To directly demonstrate that reduced NEP activity caused by antigen exposure resulted in decreased SP inactivation, we identified intact SP and its hydrolysis fragments in effluent from tracheally perfused lungs injected with ${ }^{3} \mathrm{H}-\mathrm{SP}$. In control lungs we identified intact SP and SP fragments (SP 1-6, SP 1-7, SP 1-9; Fig. $4 B$ ). These sites correspond to the known SP hydrolysis sites of NEP. We have previously shown that inhibitors of NEP do not eliminate these fragments, suggesting that enzymes other than NEP cleave and inactivate SP. However, since NEP inhibition alone enhances the contractile effects of SP in this system it is the predominant enzyme limiting SP bronchoconstriction in normal lungs. In lungs removed from animals repeatedly exposed to antigen, a greater quantity of intact SP and a smaller quantity of the NEP hydrolysis product SP 1-7 was recovered (Fig. $4 A$ ). These findings directly demonstrate that antigen-exposed lungs are more sensitive than saline-exposed lungs to the contractile effects of SP and that SP is less efficiently inactivated by NEP in these inflamed lungs. We cannot exclude the possibility that other enzymes with the capacity to cleave SP, such as mast cell chymase might have altered activity in antigen exposed lungs, however the loss of activity of the specific NEP inhibitor in antigen exposed lungs indicates that these lungs are deficient in the predominant enzyme that limits SP bronchoconstriction in normal lungs. The decreased NEP activity we observed could reflect oxidative loss of enzyme activity, loss of activity due to cellular internalization of NEP or decreased activity due to decreased transcription or translation of NEP mRNA.

In contrast to the enhanced effects of SP observed in lungs from antigen-exposed animals, these lungs were resistant to the relaxant effects of tracheally injected VIP. Furthermore, the 
resistance to the pulmonary relaxant effects of VIP in lungs from antigen-exposed animals is not a generalized inability to respond to inhibitory agonists, as isoproterenol-induced pulmonary relaxation was not altered (Fig. 5). Instead, our data suggest that the decreased sensitivity to VIP is the result of increased degradation of this peptide, probably by a tryptic enzyme. It is now established that the physiological effects of VIP in this model of airway peptide delivery are limited by enzymatic inactivation in the microenvironment by NEP and a tryptic enzyme, which are equally important and physiologically competitive $(11,17,33)$. To determine which, if any, enzyme systems were altered by airway inflammation, we compared the quantity of intact ${ }^{125} \mathrm{I}$-VIP and its radiolabeled hydrolysis fragments (VIP 1-14, VIP 4, 5-14) recovered from the effluent of lungs from antigen- or saline-exposed animals. The VIP cleavage product profile of saline-exposed lungs demonstrated VIP hydrolysis fragments consistent with inactivation by NEP and a tryptic enzyme and was similar to the profile we previously reported for normal lungs (Fig. $6 A$ ) $(11)$. In the effluent of lungs from antigen-exposed animals, no hydrolysis products consistent with NEP activity were recovered (Fig. 6 $B$ ), thus providing further evidence of decreased NEP activity in inflamed lungs. Furthermore, less intact VIP 1-28 was recovered from the lung effluent of antigen-exposed than saline-exposed animals thereby supporting the hypothesis that antigenexposed, inflamed lungs degrade VIP in an abnormally efficient manner.

The effluent of lungs from antigen-exposed animals contained greater amounts of radiolabel that coeluted with VIP 1-14 than the effluent of saline-exposed lungs, an indication that these lungs possess enhanced activity of an enzyme that hydrolyses VIP at the 14-15 bond. We have previously shown that VIP 1-14 arises from proteolytic cleavage by a tryptic enzyme that can be inhibited by SBTI (11); this pattern of cleavage is what we would expect from the action of a rodent mast cell tryptase on VIP $(34,35)$. Since the tryptic enzyme that inactivated VIP in normal lungs was sensitive to SBTI, we examined the effects of SBTI in lungs removed from animals repeatedly exposed to antigen and in control lungs. We confirmed our previous findings by demonstrating that SBTI had no effect on VIP-induced pulmonary relaxation in lungs from saline-exposed animals. In contrast, SBTI alone enhanced the relaxant response to VIP in lungs from antigen-exposed animals (Fig. 7) and was associated with enhanced recovery of active, unhydrolized VIP and diminished recovery of inactive VIP 1-14. These data implicate a tryptic enzyme as the primary modulator of VIP relaxant activity in inflamed lungs.

Antigen exposure is known to activate mast cells and liberate mast cell proteases into the extracellular microenvironment $(36,37)$. In this study, allergic inflammation was associated with histologic evidence of mast cell degranulation. The facts that the tryptic enzyme regulating VIP-induced relaxation had a hydrolysis profile for VIP identical to that of mast cell tryptase, was inhibited by inhibitors of serine proteases, and was strikingly more active in lungs removed from animals with evidence of mast cell degranulation support the hypothesis that the enzyme regulating the physiological activity of VIP in inflamed lungs is a mast cell protease. It is possible that the failure of SBTI to augment VIP-induced pulmonary relaxation above that seen in untreated control lungs reflects the inability of SBTI to completely prevent VIP hydrolysis. It is also possible that the VIP binding activity of the VIP receptor is reduced by tryptic hydrolysis in inflamed lungs (38). Our data do not allow us to distinguish between these possible mechanisms of impaired VIP effect.

Our data provide a possible mechanism for enhanced nonspecific airway responsiveness in inflamed lungs. We and others have previously shown that a variety of bronchoactive mediators release tachykinins from guinea pig lungs (39-42). Therefore, the loss of NEP activity observed in antigen-exposed lungs could result in an enhanced response to tachykinins released by other bronchoactive mediators. These data agree with the observation that enhanced methacholine (43) and antigen (44) responses in lungs of animals repeatedly exposed to antigen can be diminished by depletion of SP with capsaicin pretreatment. However, our data extend this mechanism by demonstrating that inflamed lungs are also resistant to the relaxant and likely homeostatic effects of VIP. Therefore, chronic inflammation modulates the microenvironment in two ways: it enhances the prophlogistic effects of peptides degraded by NEP, such as SP, while it diminishes the homeostatic effects of VIP. Thus, through disruption of the balance between contractile and relaxant neuropeptides in the lung, inflammation can promote nonspecific airway hyperresponsiveness.

\section{References}

1. Barnes, P. J. 1989. A new approach to the treatment of asthma. $N$. Engl. J. Med. 321:1517-1527.

2. Mcfadden, E. R., and I. A. Gilbert. 1992. Medical Progress-Asthma. N. Engl. J. Med. 327:1928-1937.

3. Nadel, J. A., and D. B. Borson. 1991. Modulation of neurogenic inflammation by neutral endopeptidase. Am. Rev. Respir. Dis. 143:S33-S36.

4. Barnes, P. J. 1991. Neurogenic inflammation in airways. Int. Arch. Allergy Appl. Immunol. 94:303-309.

5. Bertrand, C., P. Geppetti, J. Baker, I. Yamawaki, and J. A. Nadel. 1993. Role of neurogenic inflammation in antigen-induced vascular extravasation in guinea pig trachea. J. Immunol. 150:1479-1485.

6. Boomsma, J. D., and S. I. Said. 1992. The Role of Neuropeptides in Asthma. Chest. 101:S389-S392.

7. Djokic, T. D., K. Sekizawa, D. B. Borson, and J. A. Nadel. 1989. Neutral endopeptidase inhibitors potentiate substance P-induced contraction in gut smooth muscle. Am. J. Physiol. 256:G39-G43.

8. Drazen, J. M., S. A. Shore, and N. P. Gerard. 1989. Substance P-induced effects in guinea pig lungs: effects of thiorphan and captopril. J. Appl. Physiol. 66:1364-1372.

9. Martins, M. A., S. A. Shore, N. P. Gerard, C. Gerard, and J. M. Drazen. 1990. Peptidase modulation of the pulmonary effects of tachykinins in tracheal superfused guinea pig lungs. J. Clin. Invest. 85:170-176.

10. Lotvall, J. O., B. E. Skoogh, P. J. Barnes, and K. F. Chung. 1990. Effects of aerosolised substance $P$ on lung resistance in guinea-pigs: a comparison between inhibition of neutral endopeptidase and angiotensin-converting enzyme. $B r . J$. Pharmacol. 100:69-72.

11. Lilly, C. M., M. A. Martins, and J. M. Drazen. 1993. Peptidase modulation of vasoactive intestinal peptide pulmonary relaxation in tracheal superfused guinea pig lungs. J. Clin. Invest. 91:235-243.

12. Hutson, P. A., M. K. Church, T. P. Clay, P. Miller, and S. T. Holgate. 1988. Early and late phase bronchoconstriction after allergen challenge of nonanesthetized guinea pigs. I. The association of disordered airway physiology to leukocyte infiltration. Am. Rev. Respir. Dis. 137:548-557.

13. Hutson, P. A., J. G. Varley, S. Sanjar, M. Kings, S. T. Holgate, and M. K. Church. 1990. Evidence that neutrophils do not participate in the late-phase airway response provoked by ovalbumin inhalation in conscious, sensitized guinea pigs. Am. Rev. Respir. Dis. 141:535-539.

14. Martin, J. L., K. Rose, G. J. Hughes, and P. J. Magistretti. 1986. [mono[125I] iodo-Tyr 10,Met017]-vasoactive intestinal polypeptide. Preparation, characterization, and use for radioimmunoassay and receptor binding. $J$. Biol. Chem. 261:5320-5327.

15. Thompson, J. E., and D. Sheppard. 1988. Phosphoramidon potentiates the increase in lung resistance mediated by tachykinins in guinea pigs. Am. Rev. Respir. Dis. 137:337-340.

16. Shore, S. A., M. A. Martins, and J. M. Drazen. 1992. Effect of the NEP inhibitor SCH32615 on airway responses to intravenous substance-P in guinea pigs. J. Appl. Physiol. 73:1847-1853. 
17. Thompson, D. C., L. Diamond, and R. J. Altiere. 1990. Enzymatic modulation of vasoactive intestinal peptide and nonadrenergic noncholinergic inhibitory responses in guinea pig tracheae. Am. Rev. Respir. Dis. 142:1119-1123.

18. Warner, E. A., R. D. Krell, and C. K. Buckner. 1990. Pharmacologic studies on the differential influence of inhibitors of neutral endopeptidase on nonadrenergic, noncholinergic contractile responses of the guinea pig isolated hilar bronchus to transmural electrical stimulation and exogenously applied tachykinins. J. Pharmacol. Exp. Ther. 254:824-830.

19. Djokic, T. D., J. A. Nadel, D. J. Dusser, K. Sekizawa, P. D. Graf, and D. B. Borson. 1989. Inhibitors of neutral endopeptidase potentiate electrically and capsaicin-induced noncholinergic contraction in guinea pig bronchi. J. Pharmacol. Exp. Ther. 248:7-11.

20. Murlas, C. G., T. P. Murphy, and V. Chodimella. 1990. O3-induced mucosa-linked airway muscle hyperresponsiveness in the guinea pig. J. Appl. Physiol. 69:7-13.

21. Lang, Z., and C. G. Murlas. 1992. Neutral endopeptidase of a human airway epithelial cell line recovers after hypochlorous acid exposure: dexamethasone accelerates this by stimulating neutral endopeptidase mRNA synthesis. $\mathrm{Am}$. J. Respir. Cell Mol. Biol. 7:300-306.

22. Dusser, D. J., E. Umeno, P. D. Graf, T. Djokic, D. B. Borson, and J. A. Nadel. 1988. Airway neutral endopeptidase-like enzyme modulates tachykinininduced bronchoconstriction in vivo. J. Appl. Physiol. 65:2585-2591.

23. Shore, S. A., N. P. Stimler-Gerard, S. R. Coats, and J. M. Drazen. 1988 Substance P-induced bronchoconstriction in the guinea pig. Enhancement by inhibitors of neutral metalloendopeptidase and angiotensin-converting enzyme. Am. Rev. Respir. Dis. 137:331-336.

24. Stimler-Gerard, N. P. 1987. Neutral endopeptidase-like enzyme controls the contractile activity of substance $\mathrm{P}$ in guinea pig lung. J. Clin. Invest. 79:18191825.

25. Honda, I., H. Kohrogi, T. Yamaguchi, M. Ando, and S. Araki. 1991. Enkephalinase inhibitor potentiates substance P-Induced and capsaicin-induced bronchial smooth muscle contractions in humans. Am. Rev. Respir. Dis 143:1416-1418.

26. Sekizawa, K., J. Tamaoki, J. A. Nadel, and D. B. Borson. 1987. Enkephalinase inhibitor potentiates substance P- and electrically induced contraction in ferret trachea. J. Appl. Physiol. 63:1401-1405.

27. Sheppard, D., J. E. Thompson, L. Scypinski, D. Dusser, J. A. Nadel, and D. B. Borson. 1988. Toluene diisocyanate increases airway responsiveness to substance $P$ and decreases airway neutral endopeptidase. J. Clin. Invest. 81:1111-1115.

28. Dusser, D. J., T. D. Djokic, D. B. Borson, and J. A. Nadel. 1989. Cigarette smoke induces bronchoconstrictor hyperresponsiveness to substance $P$ and inactivates airway neutral endopeptidase in the guinea pig. Possible role of free radicals. J. Clin. Invest. 84:900-906.

29. Lang, Z. H., and C. G. Murlas. 1991. HOCl exposure of a human airway epithelial cell line decreases its plasma membrane neutral endopeptidase. Lung. 169:311-323.
30. Murlas, C. G., Z. Lang, G. J. Williams, and V. Chodimella. 1992. Aerosolized neutral endopeptidase reverses ozone-induced airway hyperreactivity to substance P. J. Appl. Physiol. 72:1133-1141.

31. Dusser, D. J., D. B. Jacoby, T. D. Djokic, I. Rubinstein, D. B. Borson, and J. A. Nadel. 1989. Virus induces airway hyperresponsiveness to tachykinins: role of neutral endopeptidase. J. Appl. Physiol. 67:1504-1511.

32. Borson, D. B., J. J. Brokaw, K. Sekizawa, D. M. Mcdonald, and J. A. Nadel. 1989. Neutral endopeptidase and neurogenic inflammation in rats with respiratory infections. J. Appl. Physiol. 66:2653-2658.

33. Tam, E. K., G. M. Franconi, J. A. Nadel, and G. H. Caughey. 1990 Protease inhibitors potentiate smooth muscle relaxation induced by vasoactive intestinal peptide in isolated human bronchi. Am. J. Respir. Cell Mol. Biol. 2:449-452.

34. Caughey, G. H., F. Leidig, N. F. Viro, and J. A. Nadel. 1988. Substance P and vasoactive intestinal peptide degradation by mast cell tryptase and chymase. J. Pharmacol. Exp. Ther. 244:133-137.

35. Katunuma, N., N. Fukusen, and H. Kido. 1986. Biological functions of serine proteases in the granules of rat mast cells. Adv. Enzyme Regul. 25:241-255.

36. Nadel, J. A., and G. H. Caughey. 1989. Roles of mast cell proteases in airways. Chest. 95:1328-1330.

37. Nadel, J. A. 1992. Biologic effects of mast cell enzymes. Am. Rev. Respir Dis. 145:S37-S41.

38. Paul, S., and S. I. Said. 1987. Characterization of receptors for vasoactive intestinal peptide solubilized from the lung. J. Biol. Chem. 262:158-162.

39. Martins, M. A., S. A. Shore, and J. M. Drazen. 1991. Release of tachykinins by histamine, methacholine, PAF, LTD4, and substance-P from guinea pig lungs. Am. J. Physiol. 261:L449-L455.

40. Saria, A., C. R. Martling, Z. Yan, E. Theodorsson-Norheim, R. Gamse, and J. M. Lundberg. 1988. Release of multiple tachykinins from capsaicin-sensitive sensory nerves in the lung by bradykinin, histamine, dimethylphenyl piperazinium, and vagal nerve stimulation. Am. Rev. Respir. Dis. 137:1330-1335.

41. Maggi, C. A., R. Patacchini, F. Perretti, S. Meini, S. Manzini, P. Santicioli, E. Del Bianco, and A. Meli. 1990. The effect of thiorphan and epithelium removal on contractions and tachykinin release produced by activation of capsaicin-sensitive afferents in the guinea-pig isolated bronchus. Naunyn-Schmiedeberg's Arch. Pharmacol. 341:74-79.

42. Manzini, S., and S. Meini. 1991. Involvement of capsaicin-sensitive nerves in the bronchomotor effects of arachidonic acid and melittin-a possible role for lipoxin-A4. Br. J. Pharmacol. 103:1027-1032.

43. Matsuse, T., R. J. Thomson, X. R. Chen, H. Salari, and R. R. Schellenberg. 1991. Capsaicin inhibits airway hyperresponsiveness but not lipoxygenase activity or eosinophilia after repeated aerosolized antigen in guinea pigs. Am. Rev. Respir. Dis. 144:368-372.

44. Manzini, S., C. A. Maggi, P. Geppetti, and C. Bacciarelli. 1987. Capsaicin desensitization protects from antigen-induced bronchospasm in conscious guinea-pigs. Eur. J. Pharmacol. 138:307-308. 